\title{
UNIMODAL POLYNOMIALS ARISING FROM SYMMETRIC FUNCTIONS
}

\author{
FRANCESCO BRENTI
}

(Communicated by Thomas H. Brylawski)

\begin{abstract}
We present a general result that, using the theory of symmetric functions, produces several new classes of symmetric unimodal polynomials. The result has applications to enumerative combinatorics including the proof of a conjecture by R. Stanley.
\end{abstract}

\section{INTRODUCTION}

A sequence of real numbers $a_{0}, a_{1}, \ldots, a_{d}$ is said to be symmetric if $a_{i}=$ $a_{d-i}$ for $i=0, \ldots,\left\lfloor\frac{d}{2}\right\rfloor$ and is said to be unimodal if there exists an index $0 \leq j \leq d$ such that $a_{1} \leq a_{2} \leq \cdots \leq a_{j-1} \leq a_{j} \geq a_{j+1} \geq \cdots \geq a_{d}$. A polynomial $\sum_{i=0}^{d} a_{i} x^{i} \in \mathbf{R}[x]$ is called symmetric (respectively, unimodal) if the sequence $\left\{a_{0}, \ldots, a_{d}\right\}$ has the corresponding property.

Unimodal polynomials arise often in combinatorics, geometry, and algebra and have been the subject of considerable research in recent years. Many different fields of mathematics have been used to prove that certain families of polynomials are unimodal such as, for example, classical analysis (see, e.g., [14, $22])$, linear algebra (see, e.g., $[10,11])$, the representation theory of Lie algebras and superalgebras (see, e.g., $[12,16,17])$, algebraic geometry (see, e.g., $[19,20])$, the theory of total positivity (see, e.g., $[2,3]$ ), the theory of symmetric functions (see, e.g., [4]), as well as the use of bijections and injections (see, e.g., [13]). We refer the reader to [20] for an excellent survey of many of these techniques and further references.

In this paper we also use the theory of symmetric functions to prove a general result (Theorem 2, below) that produces many new infinite families of symmetric unimodal polynomials. The result has a wide applicability, in particular, a conjecture and a result of $\mathrm{R}$. Stanley both follow as specializations of it.

For brevity reasons, we will call a nonzero polynomial a $\Lambda$-polynomial if it has nonnegative coefficients and is both symmetric and unimodal. Note that we require a $\Lambda$-polynomial to be nonzero. If $p(x)$ is a $\Lambda$-polynomial then

Received by the editors February 20, 1989 and, in revised form, April 18, 1989.

1980 Mathematics Subject Classification (1985 Revision). Primary 05A15, 05A20; Secondary $20 \mathrm{C} 30$. 
there is a unique $n \in \mathbf{N}$ such that $x^{n} p(1 / x)=p(x)$. We call the number $n / 2$ the center of symmetry of $p(x)$, and we write $C(p)=n / 2$. So, for example, $C\left(x^{2}+3 x^{3}+x^{4}\right)=3$ and $C(1+x)=1 / 2$. An elementary, though crucial property of $\Lambda$-polynomials, which will be used repeatedly in this paper, is the following.

Theorem 1. Let $p(x)$ and $q(x)$ be two $\Lambda$-polynomials. Then $p(x) q(x)$ is a $\Lambda$-polynomial and $C(p q)=C(p)+C(q)$.

Theorem 1 is well known and a proof of it can be found, e.g., in [20], Proposition 1.2 , or in [1].

\section{THE MAIN RESULT}

Let $x_{1}, x_{2}, x_{3}, \ldots$ be independent variables, and let $R\left[\left[x_{1}, x_{2}, \ldots\right]\right]$ be the ring of formal power series in $x_{1}, x_{2}, x_{3}, \ldots$ with coefficients in $R$, where $R$ is a commutative ring with identity (we refer the reader to [8] for the definition and the basic properties of $\left.R\left[\left[x_{1}, x_{2}, \ldots\right]\right]\right)$. An element $p \in$ $R\left[\left[x_{1}, x_{2}, \ldots\right]\right]$ is called symmetric if $p\left(x_{\sigma(1)}, x_{\sigma(2)}, \ldots\right)=p\left(x_{1}, x_{2}, \ldots\right)$ for all bijections $\sigma: \mathbf{P} \rightarrow \mathbf{P}$ (where $\mathbf{P} \stackrel{\text { def }}{=}\{1,2,3, \ldots\}$ ), and is said to be bounded if there is a constant $M$ such that all the monomials appearing in $p$ have degree $\leq M$. We let

$$
\hat{\Lambda}_{R} \stackrel{\text { def }}{=}\left\{p \in R\left[\left[x_{1}, x_{2}, \ldots\right]\right] ; p \text { is symmetric }\right\}
$$

and

$$
\Lambda_{R} \stackrel{\text { def }}{=}\left\{p \in \hat{\Lambda}_{R}, p \text { is bounded }\right\} ;
$$

then $\Lambda_{R}$ and $\hat{\Lambda}_{R}$ are subrings of $R\left[\left[x_{1}, x_{2}, \ldots\right]\right]$ and $\Lambda_{R} \subset \hat{\Lambda}_{R}$. We call $\hat{\Lambda}_{R}$ the ring of symmetric formal power series and $\Lambda_{R}$ the ring of symmetric functions, with coefficients in $R$. Note that $\Lambda_{R} \underset{\neq}{\subset} \hat{\Lambda}_{R}$ since, for example, $\prod_{i \geq 1}\left(1+x_{i}\right) \in \hat{\Lambda}_{R} \backslash \Lambda_{R}$.

We will follow Chapter I of [9] for symmetric function notation and terminology. In particular, we will denote by $s_{\lambda}$ (respectively, $h_{\lambda}, e_{\lambda}, m_{\lambda}$, and $p_{\lambda}$ ) the Schur (respectively, complete homogeneous, elementary, monomial, and power sum) symmetric functions, associated to the partition $\lambda$. We also denote by $\mathscr{P}$ the set all partitions, and by $\Lambda_{\mathrm{Q}}^{n}$ the subspace of all elements of $\Lambda_{\mathbf{Q}}$ that are homogeneous of degree $n$. If $\lambda=\left(\lambda_{1} \geq \lambda_{2} \geq \cdots \geq \lambda_{l}>0\right) \in \mathscr{P}$ then let $|\lambda| \stackrel{\text { def }}{=} \sum_{i=1}^{l} \lambda_{i}$, and also write $\lambda \vdash n$ if $n=|\lambda|$. We call a basis $\left\{a_{\lambda}\right\}_{\lambda \in \mathscr{P}}$ of $\Lambda_{\mathbf{Q}}$ standard if, for all $n \in \mathbf{N},\left\{a_{\lambda}: \lambda \vdash n\right\}$ is a basis of $\Lambda_{\mathbf{Q}}^{n}$. Note that a standard basis $\left\{a_{\lambda}\right\}_{\lambda \in \mathscr{S} \rho}$ is homogeneous and that $\operatorname{deg}\left(a_{\lambda}\right)=|\lambda|$.

We now come to the crucial definition of this paper. We say that an ordered triple $\left(\left\{a_{\lambda}\right\}_{\lambda \in \mathscr{P}},\left\{b_{\lambda}\right\}_{\lambda \in \mathscr{P}},\left\{c_{\lambda}\right\}_{\lambda \in \mathscr{P}}\right)$ of standard bases of $\Lambda_{\mathbf{Q}}$ is compatible if:

(i) if $c_{\lambda}=\sum_{\mu} f_{\lambda \mu} a_{\mu}$ then $f_{\lambda \mu} \geq 0$, for all $\lambda, \mu \in \mathscr{P}$;

(ii) if $a_{\lambda} b_{\mu}=\sum_{\nu} g_{\lambda \mu}^{\nu} a_{\nu}$ then $g_{\lambda \mu}^{\nu} \geq 0$, for all $\lambda, \mu, \nu \in \mathscr{P}$. 
Conditions (i) and (ii) above are very mild and often automatically satisfied. In fact, we have the following result.

Proposition 1. The ordered triple $\left(\left\{a_{\lambda}\right\}_{\lambda \in \mathscr{P}},\left\{b_{\lambda}\right\}_{\lambda \in \mathscr{P}},\left\{c_{\lambda}\right\}_{\lambda \in \mathscr{P}}\right)$ is compatible in each of the following cases:

(i) $a_{\lambda}=b_{\lambda}=c_{\lambda} \in\left\{m_{\lambda}, e_{\lambda}, h_{\lambda}, s_{\lambda}, p_{\lambda}\right\}$;

(ii) $a_{\lambda}=m_{\lambda}$, and $b_{\lambda}=c_{\lambda} \in\left\{m_{\lambda}, e_{\lambda}, h_{\lambda}, s_{\lambda}, p_{\lambda}\right\}$;

(iii) $a_{\lambda}=p_{\lambda}$, and $b_{\lambda}, c_{\lambda} \in\left\{p_{\lambda}, h_{\lambda}\right\}$;

(iv) $a_{\lambda}=s_{\lambda}$, and $b_{\lambda}, c_{\lambda} \in\left\{e_{\lambda}, h_{\lambda}, s_{\lambda}\right\}$.

The proof of the preceding Proposition follows immediately from the results in Chapter 1, $\S 6$ of [9] and from our definitions and is therefore omitted.

We are now in a position to state and prove the main result of this paper.

Theorem 2. Let $\left\{r_{\mu}(q)\right\}_{\mu \in S},\left\{u_{\nu}(q)\right\}_{\nu \in T}$ be two families of $\Lambda$-polynomials, where $S, T \subseteq \mathscr{P}$ and $(0) \notin S$. Suppose that there exists $n, k \in \mathbf{Z}$ such that

$$
C\left(r_{\mu}\right)=\frac{n|\mu|}{2}, \quad C\left(u_{\nu}\right)=\frac{n|\nu|+k}{2},
$$

for all $\mu \in S, \nu \in T$. For $\rho \in \mathscr{P}$, define a polynomial $R_{\rho}(q)$ by

$$
\sum_{\rho \in \mathscr{P}} R_{\rho}(q) a_{\rho}=\frac{\sum_{\nu \in T} u_{\nu}(q) c_{\nu}}{1-\sum_{\mu \in S} r_{\mu}(q) b_{\mu}}
$$

where $\left(\left\{a_{\lambda}\right\}_{\lambda \in \mathscr{P}},\left\{b_{\lambda}\right\}_{\lambda \in \mathscr{P}},\left\{c_{\lambda}\right\}_{\lambda \in \mathscr{P}}\right)$ is a compatible triple of standard bases of $\Lambda_{\mathbf{Q}}$. Then $R_{\rho}(q)$ is a $\Lambda$-polynomial and $C\left(R_{\rho}\right)=(n|\rho|+k) / 2$.

Proof. Note first that the symmetric formal power series $1-\sum_{\mu \in S} r_{\mu}(q) b_{\mu}$ is an invertible element of $\mathbf{Q}[q]\left[\left[x_{1}, x_{2}, \ldots\right]\right]$ and that, therefore, the expression on the RHS of (1) is a well defined element of $\hat{\Lambda}_{\mathrm{Q}[q]}$. Now let

$$
c_{\nu}=\sum_{\lambda \in \mathscr{P}} f_{\nu \lambda} a_{\lambda}
$$

and

$$
a_{\rho} b_{\mu}=\sum_{\lambda} g_{\rho \mu}^{\lambda} a_{\lambda}
$$

From our hypotheses and definitions it then follows that $f_{\nu \lambda}=0$ unless $|\nu|=$ $|\lambda|, g_{\rho \mu}^{\lambda}=0$ unless $|\lambda|=|\rho|+|\mu|$, and

$$
f_{\nu \lambda} \geq 0, \quad g_{\rho \mu}^{\lambda} \geq 0
$$

for all $\nu, \lambda, \rho, \mu \in \mathscr{P}$. Now rewrite (1) as

$$
\sum_{\rho \in \mathscr{P}} R_{\rho}(q) a_{\rho}-\sum_{\rho \in \mathscr{P}} \sum_{\mu \in S} R_{\rho}(q) r_{\mu}(q) a_{\rho} b_{\mu}=\sum_{\nu \in T} u_{\nu}(q) c_{\nu} .
$$


Substituting (2) and (3) in (4) and then equating the coefficients of $a_{\lambda}$ on both sides obtains

$$
\left\{\begin{array}{l}
R_{\lambda}(q)=\sum_{\rho \in \mathscr{P}} \sum_{\mu \in S} R_{\rho}(q) r_{\mu}(q) g_{\rho \mu}^{\lambda}+\sum_{\nu \in T} u_{\nu}(q) f_{\nu \lambda} \quad \text { if } \lambda \neq 0, \\
R_{(0)}(q)=f_{(0)(0)} u_{(0)}(q) .
\end{array}\right.
$$

Proceeding by induction on $|\lambda|$, the thesis is clearly true if $|\lambda|=0$. So fix $\lambda \in \mathscr{P}$ with $|\lambda| \geq 1$ and assume that the thesis holds for all partitions such that $|\rho|<$ $|\lambda|$. Since $(0) \notin S,|\rho|<|\lambda|$ and hence, by our induction hypothesis, $R_{\rho}(x)$ is a $\Lambda$-polynomial and $C\left(R_{\rho}\right)=(n|\rho|+k) / 2$, for all partitions $\rho$ appearing in the first sum on the RHS of (5). But, by hypothesis, $r_{\mu}(x)$ is a $\Lambda$-polynomial and $C\left(r_{\mu}\right)=n|\mu| / 2$ for all $\mu \in S$. Therefore, by Theorem $1, R_{\rho}(x) r_{\mu}(x)$ is a $\Lambda$-polynomial and

$$
\begin{aligned}
C\left(R_{\rho} r_{\mu}\right) & =\frac{n(|\rho|+|\mu|)+k}{2} \\
& =\frac{n|\lambda|+k}{2}
\end{aligned}
$$

for all $\rho$ and $\mu$ appearing in the first sum on the RHS of (5). On the other hand, by our assumptions, all polynomials $u_{\nu}(x) f_{\nu \lambda}$ appearing in the second sum on the RHS of (5) are also $\Lambda$-polynomials and

$$
\begin{aligned}
C\left(u_{\nu}(x) f_{\nu \lambda}\right) & =\frac{n|\nu|+k}{2} \\
& =\frac{n|\lambda|+k}{2} .
\end{aligned}
$$

So all polynomials on the RHS of (5) are $\Lambda$-polynomials with center of symmetry equal to $(n|\lambda|+k) / 2$. Therefore their sum, $R_{\lambda}(x)$, is also a $\Lambda$-polynomial with the same center of symmetry, as desired. This concludes the induction step and hence the proof.

It may seem that Theorem 2 should be of limited use because it produces only one sequence of $\Lambda$-polynomials starting with $t w o$ such sequences. However, as will be seen in the next section, in many applications the sequence $\left\{R_{\lambda}(q)\right\}_{\lambda \in \mathscr{S}}$ is much more complicated than the two starting sequences $\left\{u_{\nu}(q)\right\}_{\nu \in T}$ and $\left\{r_{\mu}(q)\right\}_{\mu \in S}$.

\section{Applications}

Our first application of Theorem 2 is the following.

Proposition 2. For each partition $\lambda$ define a polynomial $R_{\lambda}(q)$ by

$$
\sum_{\lambda} R_{\lambda}(q) s_{\lambda}=\frac{1}{1-\sum_{k \geq 2}\left(q+q^{2}+\cdots+q^{k-1}\right) s_{k}}
$$

Then $R_{\lambda}(q)$ is a $\Lambda$-polynomial and $C\left(R_{\lambda}\right)=|\lambda| / 2$. 
Proof. Take $a_{\lambda}=b_{\lambda}=c_{\lambda}=s_{\lambda}$ for $\lambda \in \mathscr{P}$ and let $S \stackrel{\text { def }}{=}\{(k): k \geq 2\}, r_{\mu}(x) \stackrel{\text { def }}{=}$ $q+q^{2}+\cdots+q^{|\mu|-1}$ if $\mu \in S, T \stackrel{\text { def }}{=}\{(0)\}, \mu_{(0)}(x) \stackrel{\text { def }}{=} 1$. Then all the hypotheses of Theorem 2 are satisfied with $n=1$ and $k=0$, and the result follows.

Proposition 2 verifies a conjecture of R. Stanley (see the remarks preceding Proposition 7.8 in [20]).

Our second application is closely related to the previous one.

Proposition 3. For each partition $\lambda$, define a polynomial $T_{\lambda}(q)$ by

$$
\sum_{\lambda} T_{\lambda}(q) s_{\lambda}=\frac{\sum_{k \geq 1}\left(1+q+\cdots+q^{k-1}\right) s_{k}}{1-\sum_{k \geq 2}\left(q+q^{2}+\cdots+q^{k-1}\right) s_{k}}
$$

Then $T_{\lambda}(q)$ is a $\Lambda$-polynomial and $C\left(T_{\lambda}\right)=(|\lambda|-1) / 2$.

Proof. Take $a_{\lambda}, b_{\lambda}, c_{\lambda}, S$ and $r_{\mu}(q)$ as in the proof of Proposition 2 and let $T \stackrel{\text { def }}{=}\{(k): k \geq 1\}, u_{\nu}(q) \stackrel{\text { def }}{=} 1+q+q^{2}+\cdots+q^{|\nu|-1}$ if $\nu \in T$. Then all the hypotheses of Theorem 2 are satisfied with $n=1$ and $k=-1$ and the result follows.

Proposition 3 is equivalent to a result of R. Stanley (see Proposition 7.7 in [20]). However, our proof only uses standard results from the theory of symmetric functions while the sketch of proof given in [20] uses techniques from the theory of representations of the symmetric group and from algebraic geometry. To see that the two results are equivalent just observe that

$$
\frac{\sum_{k \geq 1}\left(1+q+\cdots+q^{k-1}\right) s_{k}}{1-\sum_{k \geq 2}\left(q+q^{2}+\cdots+q^{k-1}\right) s_{k}}+1=\frac{\sum_{k \geq 0} s_{k}}{1-\sum_{k \geq 2}\left(q+q^{2}+\cdots+q^{k-1}\right) s_{k}}
$$

which is the definition used in Proposition 7.7 of [20].

\section{COMBINATORIAL CONSEQUENCES}

In this section we look at some combinatorial properties of the polynomials studied in the last section. Following [7, §3], we define a map $R: \Lambda_{\mathrm{Q}[q]} \rightarrow$ $\mathbf{Q}[q][[t]]$ by

$$
R(p) \stackrel{\text { def }}{=} \sum_{n \geq 0}\left[x_{1} \cdots x_{n}\right](p) \frac{t^{n}}{n !}
$$

where $p \in \Lambda$ and $\left[x_{1} \cdots x_{n}\right](p)$ denotes the coefficient of $x_{1} \cdots x_{n}$ in $p$ (where $\prod_{i=1}^{n} x_{i} \stackrel{\text { def }}{=} 1$ if $\left.n=0\right)$. It is then easy to see that $R$ is a ring homomorphism. Also, from the combinatorial interpretation of the Schur functions (see, e.g., [9, Equation (5.12), p. 42] or [15]) it follows immediately that, for any partition $\lambda$,

$$
R\left(s_{\lambda}\right)=f^{\lambda} \frac{t^{|\lambda|}}{|\lambda| !}
$$


where $f^{\lambda}$ denotes the number of standard tableaux of shape $\lambda$ (see, e.g., [9, p. 5] for the definition of a standard tableau).

We may now prove the following result.

Proposition 4. Let $T_{\lambda}(q)$ be the polynomials defined in Proposition 3. Then, for $n \in \mathbf{P}$,

$$
\sum_{\lambda \vdash n} f^{\lambda} T_{\lambda}(q)=\frac{1}{q} A_{n}(q),
$$

where $A_{n}(q)$ is the nth Eulerian polynomial.

Proof. Multiplying both sides of (7) by $q$ and then adding 1 gives

$$
1+\sum_{\lambda \in \mathscr{P}} q T_{\lambda}(q) s_{\lambda}=\frac{1+q t+\sum_{k \geq 2} q^{k} s_{k}}{1-\sum_{k \geq 2}\left(q+q^{2}+\cdots+q^{k-1}\right) s_{k}} ;
$$

applying $R$ on both sides and using (8) and (9) we obtain

$$
\begin{aligned}
1+\sum_{\lambda \in \mathscr{P}} q T_{\lambda}(q) f^{\lambda} \frac{t^{|\lambda|}}{|\lambda| !} & =\frac{e^{q t}}{1-\frac{1}{1-q}\left(q e^{t}-q-e^{q t}+1\right)} \\
& =\frac{(1-q) e^{q t}}{e^{q t}-q e^{t}} \\
& =\sum_{n \geq 0} A_{n}(q) \frac{t^{n}}{n !},
\end{aligned}
$$

where, in the last equality, we have used a well known generating function for Eulerian polynomials (see, e.g., Equation (5i) on p. 244 of [5]). Equating the coefficients of $t^{n}$ yields (10), as desired.

Note that, by Proposition 3, the preceding result gives yet another proof of the well known fact that the Eulerian polynomials are symmetric and unimodal.

Now let $n \in \mathbf{P}$ and $\sigma \in S_{n}$ (where $S_{n}$ is the symmetric group on $n$ elements). An element $i \in[n]$ (where $[n] \stackrel{\text { def }}{=}\{1,2, \ldots, n\}$ ) is called an excedance of $\sigma$ if $\sigma(i)>i$; denote the number of excedances of $\sigma$ by $e(\sigma)$. The permutation $\sigma \in S_{n}$ is called a derangement if $\sigma(i) \neq i$ for $i=1,2, \ldots, n$ (i.e., $\sigma$ has no fixed points). We denote the set of all derangements of $S_{n}$ by $D_{n}$, we define polynomials $d_{n}(q)$ by

$$
d_{n}(q) \stackrel{\text { def }}{=} \sum_{\sigma \in D_{n}} q^{e(\sigma)}
$$

for $n \in \mathbf{P}$ (so that $d_{1}(q)=0$ ), and let $d_{0}(q) \stackrel{\text { def }}{=} 1$. Since $d_{n}(1)=\left|D_{n}\right|$ we may consider $d_{n}(q)$ as a $q$-analogue of the derangement numbers. These are different, however, from other $q$-derangement numbers that have been previously considered in the literature (see, e.g., $[6,21]$ ).

It is not hard to write down the exponential generating function for the polynomials $d_{n}(q)$. 
Proposition 5. Let $d_{n}(q)$ be the polynomials defined by (11). Then

$$
\sum_{n \geq 0} d_{n}(q) \frac{t^{n}}{n !}=\frac{1-q}{e^{t q}-q e^{t}} .
$$

Proof. It is well known (see, e.g., [18, Proposition 1.3.12]) that $A_{n}(q)=$ $\sum_{\sigma \in S_{n}} q^{e(\sigma)+1}$. Therefore, for $n \in \mathbf{P}$,

$$
\begin{aligned}
\frac{1}{q} A_{n}(q) & =\sum_{S \subseteq[n]} \sum_{\sigma \in D_{|s|}} q^{e(\sigma)} \\
& =\sum_{i=0}^{n}\left(\begin{array}{c}
n \\
i
\end{array}\right) d_{i}(q) .
\end{aligned}
$$

It follows that

$$
\begin{aligned}
q e^{t} \sum_{n \geq 0} d_{n}(q) \frac{t^{n}}{n !} & =\sum_{n \geq 0} A_{n}(q) \frac{t^{n}}{n !}+q-1 \\
& =\frac{(1-q) e^{q t}}{e^{q t}-q e^{t}}+(q-1) \\
& =\frac{(1-q) q e^{t}}{e^{t q}-q e^{t}}
\end{aligned}
$$

where in the last equality we have again used Equation (5i) on p. 244 of [5], and the proof follows.

We can now prove the following result.

Proposition 6. Let $R_{\lambda}(q)$ be the polynomials defined in Proposition 2. Then, for $n \in \mathbf{N}$,

$$
\sum_{\lambda \vdash n} f^{\lambda} R_{\lambda}(q)=d_{n}(q) .
$$

Proof. Applying the homomorphism $R$ defined by (8) to both sides of (6) yields

$$
\begin{aligned}
\sum_{\lambda \in \mathscr{D}} R_{\lambda}(q) f^{\lambda} \frac{t^{|\lambda|}}{|\lambda| !} & =\frac{1}{1-\sum_{k \geq 2}\left(q+q^{2}+\cdots+q^{k-1}\right) \frac{t^{k}}{k !}} \\
& =\frac{1-q}{e^{t q}-q e^{t}} \\
& =\sum_{n \geq 0} d_{n}(q) \frac{t^{n}}{n !},
\end{aligned}
$$

and the proof follows.

By Proposition 2, the following is an immediate consequence of the previous result. 
Corollary 1. For $n \in \mathbf{P}$, the polynomials $d_{n}(q)$ defined by (11) are symmetric and unimodal.

It would be interesting to have a combinatorial proof of this result.

Even though the techniques presented in this paper cannot be used to prove it, we feel that the following stronger statement actually holds.

Conjecture. For $n \in \mathbf{P}$, the polynomials $d_{n}(q)$ defined by (11) have only real zeros.

The conjecture has been verified for $n \leq 14$. It is possible that the techniques used in [2] and [3] may be useful in attacking the above conjecture which, in fact, is closely related to one of the conjectures appearing in $\S 3.4$ of [2].

Given the results of Propositions 4 and 6 it is natural to ask for a combinatorial interpretation of the polynomials $T_{\lambda}(q)$ and $R_{\lambda}(q)$ themselves, a problem that had already been raised in [20]. Such a combinatorial interpretation has recently been found by $\mathbf{J}$. Stembridge and will appear in a forthcoming paper of his.

\section{ACKNOWLEDGMENT}

The author would like to thank Richard Stanley for some valuable suggestions in $\S 4$.

\section{REFERENCES}

1. G. E. Andrews, $A$ theorem on reciprocal polynomials with applications to permutations and compositions, Amer. Math. Monthly 82 (1975), 830-833.

2. F. Brenti, Unimodal, log-concave and Pólya frequency sequences in combinatorics, Ph. D. thesis, June 1988, Massachusetts Institute of Technology.

3. __, Unimodal, log-concave and Pólya frequency sequences in combinatorics, Memoirs Amer. Math. Soc. 81, no. 413, 1989.

4. L. Butler, A unimodality result in the enumeration of subgroups of a finite abelian group, Proc. Amer. Math. Soc. 101 (1987), 771-775.

5. L. Comtet, Advanced combinatorics, Reidel, Dordrecht/Boston, 1974.

6. A. M. Garsia and J. Remmel, A combinatorial interpretation of q-derangement and $q$-Laguerre numbers, European J. Combin. 1 (1980), 47-59.

7. I. Gessel and G. Viennot, Determinants, paths, and plane partitions, preprint.

8. I. P. Goulden and D. M. Jackson, Combinatorial enumeration, John Wiley, New York, 1983.

9. I. G. MacDonald, Symmetric functions and Hall polynomials, Oxford University Press, New York/London, 1979.

10. R. Proctor, Representations of $\mathrm{sl}(2, C)$ on posets and the Sperner property, SIAM J. Algebraic Discrete Methods 3 (1982), 275-280.

11. Solution of two difficult combinatorial problems with linear algebra, Amer. Math. Monthly 89 (1982), 721-734.

12. __ Bruhat lattices, plane partitions generating functions, and minuscule representations, European J. Combin. 5 (1984), 331-350.

13. B. Sagan, Inductive and injective proofs of log concavity results, Discrete Math. 68 (1988), 281-292. 
14. R. Simion, A multiindexed Sturm sequence of polynomials and unimodality of certain combinatorial sequences, J. Combin. Theory Ser. A 36 (1984), 15-22.

15. R. Stanley, Theory and application of plane partitions: Part 1, Studies Applied Math. 50 (1971), 167-188.

16. __ Unimodal sequences arising from Lie algebras, in Young Day Proceedings (T. V. Narayana, R. M. Mathsen, and J. G. Williams, eds.), Dekker, New York/Basel, 1980, 127-136.

17. __ Unimodality and Lie superalgebras, Studies in Applied Math. 72 (1985), 263-281.

18. __ Enumerative combinatorics, vol. 1, Wadsworth and Brooks/Cole, Monterey, California 1986.

19. __ Generalized h-vectors, intersection cohomology of toric varieties and related results, Advanced Studies in Pure Math. 11 (1987), 187-213.

20. __ Log-concave and unimodal sequences in algebra, combinatorics and geometry, (to appear in Annals of the New York Academy of Sciences).

21. M. Wachs, On q-derangement numbers, Proc. Amer. Math. Soc. 106 (1989), 273-278.

22. D. Wagner, The partition polynomial of a finite set system, (to appear in J. Combin. Theory Ser. A).

Department of Mathematics, University of Michigan, Ann Arbor, Michigan 48109 1003 